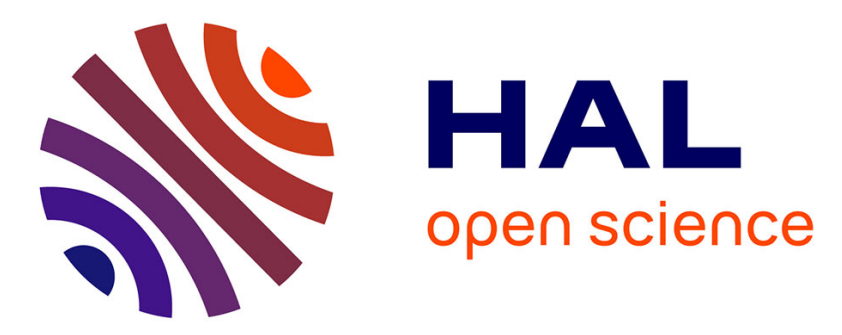

\title{
Functional ultrasound imaging of the spreading activity following optogenetic stimulation of the rat visual cortex
}

\author{
M. Provansal, G. Labernède, C. Joffrois, A. Rizkallah, R. Goulet, M. Valet,
} W. Deschamps, U. Ferrari, A. Chaffiol, D. Dalkara, et al.

\section{- To cite this version:}

M. Provansal, G. Labernède, C. Joffrois, A. Rizkallah, R. Goulet, et al.. Functional ultrasound imaging of the spreading activity following optogenetic stimulation of the rat visual cortex. Scientific Reports, 2021, 11 (1), pp.12603. 10.1038/s41598-021-91972-z . hal-03263679

\section{HAL Id: hal-03263679 \\ https://hal.sorbonne-universite.fr/hal-03263679}

Submitted on 17 Jun 2021

HAL is a multi-disciplinary open access archive for the deposit and dissemination of scientific research documents, whether they are published or not. The documents may come from teaching and research institutions in France or abroad, or from public or private research centers.
L'archive ouverte pluridisciplinaire HAL, est destinée au dépôt et à la diffusion de documents scientifiques de niveau recherche, publiés ou non, émanant des établissements d'enseignement et de recherche français ou étrangers, des laboratoires publics ou privés. 


\title{
scientific reports
}

Check for updates

\section{OPEN Functional ultrasound imaging of the spreading activity following optogenetic stimulation of the rat visual cortex}

\author{
M. Provansal ${ }^{1 凶}$, G. Labernède ${ }^{1}$, C. Joffrois ${ }^{1}$, A. Rizkallah ${ }^{1}$, R. Goulet $^{1}$, M. Valet $^{1}$, \\ W. Deschamps ${ }^{1}$, U. Ferrari ${ }^{1}$, A. Chaffiol ${ }^{1}$, D. Dalkara ${ }^{1}$, J. A. Sahel ${ }^{1,2,3}$, M. Tanter $^{4}$, S. Picaud ${ }^{1}$, \\ G. Gauvain ${ }^{1,5}$ \& F. Arcizet ${ }^{1,5}$
}

Optogenetics has revolutionized neurosciences by allowing fine control of neuronal activity. An important aspect for this control is assessing the activation and/or adjusting the stimulation, which requires imaging the entire volume of optogenetically-induced neuronal activity. An ideal technique for this aim is fUS imaging, which allows one to generate brain-wide activation maps with submesoscopic spatial resolution. However, optical stimulation of the brain with blue light might lead to non-specific activations at high irradiances. fUS imaging of optogenetic activations can be obtained at these wavelengths using lower light power $(<2 \mathrm{~mW})$ but it limits the depth of directly activatable neurons from the cortical surface. Our main goal was to report that we can detect specific optogenetic activations in V1 even in deep layers following stimulation at the cortical surface. Here, we show the possibility to detect deep optogenetic activations in anesthetized rats expressing the red-shifted opsin ChrimsonR in V1 using fUS imaging. We demonstrate the optogenetic specificity of these activations and their neuronal origin with electrophysiological recordings. Finally, we show that the optogenetic response initiated in V1 spreads to downstream (LGN) and upstream (V2) visual areas.

Optogenetics has revolutionized investigation of the central nervous system ${ }^{1-5}$ by allowing fine control of neurons in living systems. A key aspect of optogenetic investigation of neuronal circuits is the read-out of the activity since it demonstrates the efficacy of the stimulation and offers the possibility to adjust it. Ideally, the technique on which it relies should have good spatial and temporal resolutions and a spatial extent covering the whole brain.

Electrophysiological recordings can report optogenetic-induced neuronal activity with unmatched temporal resolution, but over a very small spatial area ${ }^{6}$. Besides, they need intracortical penetration. Calcium imaging is a technique that also allows a temporally fine read-out of the activity but it is spatially limited in depth due to light absorption. Conversely, Lee and colleagues have demonstrated the possibility to combine optogenetics with fMRI (a combination named ofMRI) in mice ${ }^{7}$, opening up a new horizon for mapping genetically specified neural circuits. Since then, ofMRI has been applied for the investigation of different brain pathways in rodents ${ }^{8-10}$ and primates ${ }^{11}$, and for the assessment of neural transplant networks ${ }^{12}$. However, fMRI has a limited spatial resolution higher than $1 \mathrm{~mm}$ and requires complex equipments ${ }^{13}$. Like fMRI, ultrafast functional ultrasound imaging (fUS) can provide brain-sized maps of neurovascular activity changes, with a higher spatial resolution $(100 \mu \mathrm{m} \times 100 \mu \mathrm{m})$ even in deep structures (up to $1.5 \mathrm{~cm})^{14-16}$. This technique has been used to investigate sensory processing in anesthetized ${ }^{17}$, awake ${ }^{17-20}$ and asleep ${ }^{21}$ rodents, and in behaving primates ${ }^{22-24}$. fUS imaging and electrophysiological recordings can, therefore, be used to describe the dynamics of local neuronal activity accurately whilst scanning neurovascular activity over the entire brain.

Recent studies have showed some limitations of using fUS imaging as a read-out technique for cerebral activity after an optogenetic stimulation. For example, Rungta and colleagues showed that optical stimulation of the brain using blue light could generate non-specific activations in naïve mice ${ }^{25}$, at light powers higher than $2 \mathrm{~mW}$

\footnotetext{
${ }^{1}$ Sorbonne Université, INSERM, CNRS, Institut de la Vision, 75012 Paris, France. ${ }^{2}$ Department of Ophthalmology, The University of Pittsburgh School of Medicine, Pittsburgh, PA 15213, USA. ${ }^{3}$ Department of Ophthalmology and Vitreo-Retinal Diseases, Fondation Ophtalmologique Rothschild, 75012 Paris, France. ${ }^{4}$ Physics for Medicine Paris, INSERM, CNRS, Ecole Supérieure de Physique Et de Chimie Industrielles (ESPCI Paris), Paris Sciences Et Lettres (PSL) Research University, 75012 Paris, France. ${ }^{5}$ These authors contributed equally: G. Gauvain and F. Arcizet. ${ }^{\varpi}$ email: matthieu.provansal@inserm.fr
} 
(corresponding to irradiances higher than $18 \mathrm{~mW} \mathrm{~mm}^{-2}$ ), restricting the range of light powers one can use to activate cerebral structures and consequently the depth of targeted areas. Recently, one study has demonstrated the possibility to detect specific optogenetic activations following blue light illumination of the retrospenial cortex in Thy-ChR2 mice or the illumination of specific neuronal subpopulations of the superior colliculus in other transgenic lines ${ }^{19}$ using lower light power $\left(0.3 \mathrm{~mW}\right.$, corresponding to an irradiance of $\left.1.5 \mathrm{~mW} \mathrm{~mm}^{-2}\right)$ to prevent non-specific activation. Another publication from the same group used the same combination of parameters to map collicular cell-specific activation circuits and their associated behaviors ${ }^{26}$. However, optogenetic activation at such low irradiances is uncertain in neurons with lower AAV-mediated expression of microbial opsins. In addition, working with blue wavelengths limits light penetration in the brain and therefore limits the maximal depth of neurons one can directly activate from the surface with low irradiances. Conversely, red-shifted opsins offer a powerful alternative for deeper neuronal stimulation, as red light propagates deeper and can be used safely at higher intensities to activate neurons than blue light ${ }^{27-29}$. In the present study, we show that red light stimulation at the cortical surface can activate visual neurons localized in deep cortical layers without triggering a thermal hemodynamic response and toxicity. In addition, we scale the entire volume of V1 activation following a focal red light stimulation at the cortical surface. We prove the optogenetic specificity of these activations even at high irradiances and we recorded their electrophysiological correlates. We were also able to follow the propagation of this information in other visual brain structures (i.e. LGN and V2). More generally, this work shows that fUS imaging has the potential to provide a clear, brain-wide mesoscopic view of the neuronal activities resulting from optogenetic stimulation even in deep structures, such as the LGN, inaccessible to optical techniques and with a higher resolution than fMRI.

\section{Results}

fUS imaging of V1 optogenetic activation in rats. We used the optogenetic actuator ChrimsonR in the visual cortex of naive Long-Evans rats, because of its red-shifted opsin properties ${ }^{27,30,31}$. We maximized the optogenetic activation of V1 by using the CaMKII promoter to ensure expression limited to the excitatory neurons of V1. Indeed, a ubiquitous promoter might lead to the silencing of pyramidal neurons through the recruitment of inhibitory neurons. ChrimsonR was fused to the fluorescent reporter tdTomato to facilitate the visualization of transfected areas. Following preliminary screening, we used the AAV9-7m8 mutated viral capsid to express ChrimsonR in the V1 neurons of Long-Evans rats (Fig. 1a). The mean rate of neuronal transfection was $5.5 \%$ over all cortical layers (Fig. S1). ChrimsonR expression was not restricted to the soma, but spread to the axons and dendrites (Fig. 1a). We assessed the efficacy of the optogenetic stimulation of V1 cortical neurons, by using fUS imaging to measure brain activity in a large proportion of the brain: from AP $-3.5 \mathrm{~mm}$ to AP $-8 \mathrm{~mm}$, the zone in which most of the early visual system areas are located. Activity in the cortical layers was assessed following either direct stimulation of the contralateral eye with a white LED $\left(58 \mathrm{~mW} \mathrm{~cm}^{-2}\right)$, or stimulation at $595 \mathrm{~nm}$ delivered with an optic fiber placed at the surface of the transfected or non-transfected V1 areas $\left(7 \mathrm{~mW}, \sim 140 \mathrm{~mW} \mathrm{~mm}^{-2}\right.$ at the brain surface) (Fig. 1b). We chose to use durations and magnitudes of parameters similar to those previously used ${ }^{17,25}$ for stimulation of the eye or cortex $(2 \mathrm{~s}$ at $4 \mathrm{~Hz}$ or $20 \mathrm{~Hz}$ for stimulation of the eye and cortex, respectively, separated by a 13 s period of darkness. This cycle was performed 20 times). For eye stimulation (Fig. 1b, left), we first imaged V1 with a single imaging plane at AP $-7.5 \mathrm{~mm}$ and constructed an activation map including only pixels displaying significant CBV (cerebral blood volume) responses $(p<0.05$ with Bonferroni-Holm correction, Wilcoxon signed-rank test, one-tailed, relative to baseline activity). With fUS imaging, we detected strong activation in both the ipsilateral and contralateral superior colliculi (SC) (ipsilateral, $n=121 / 488$ activated pixels; contralateral, $n=134 / 421$ activated pixels), but almost no activation in the ipsilateral and contralateral V1 areas (ipsilateral, $n=8 / 634$ activated pixels; contralateral, $n=9 / 370$ activated pixels). The lack of response detected with fUS imaging in both V1 areas and the strong signal in both SC may reflect the retinotectal nature of most rodent retinal outputs ${ }^{32}$ or an effect of anesthesia ${ }^{33}$. An increase in CBV was already clearly visible on single-cycle responses (gray dashed lines), as illustrated for the significant pixel (\#14-92) in the ipsilateral V1 area (insert). Following direct stimulation of the contralateral eye, the mean response (black curve) peaked $2 \mathrm{~s}$ after the two-second stimulation represented by the patch in gray (mean: $19.8 \pm 18.3 \%$ ). For cortical stimulation (Fig. 1b, center), we observed a broad activation of the ipsilateral V1 area $(n=310 / 634$ activated pixels). The activation spread out of the V1 area at each border (medial and ventral) with V1 projections onto other visual areas. Single-cycle responses of the same example pixel showed larger, less variable increases in CBV variation than for stimulation of the contralateral eye (optogenetic, mean: $65.8 \pm 32.6 \%, p<0.0001$, Mann-Whitney). A previous study ${ }^{25}$ showed that blue light delivery to the brain could itself generate non-specific changes in CBV at light powers higher than $2 \mathrm{~mW}$ (irradiances higher than $18 \mathrm{~mW} \mathrm{~mm}^{-2}$ ). We therefore performed a control stimulation by locating the optic fiber at the surface of the non-injected V1 area, which did not express ChrimsonR-tdT (Fig. 1b, right). We used the same light stimulation parameters for this control as before. We detected no significant CBV responses in the non-injected V1 area under such optogenetic stimulation conditions.

We further characterized the V1 activation volume generated by contralateral eye stimulation or by stimulation of the transfected V1 area, by imaging all the planes containing V1 (from AP -6 to $-8 \mathrm{~mm}$, Fig. 1c-d). As shown in Fig. 1b, direct eye stimulation induced CBV responses mostly in the SC areas, but very little activation was observed in the two V1 areas (mean: $0.05 \pm 0.12 \%$ ). Strikingly for the same animal shown (as shown in Fig. 1c), direct optogenetic stimulation of the injected V1 area generated significantly stronger CBV responses, with a mean active volume of $37 \%$ (range: 0.5 to $75.9 \%$ ). The percentage mean active injected V1 area, over all animals, was higher for optogenetic cortical stimulation than for direct eye stimulation (optogenetic, mean: $16.2 \pm 17.8 \%$, Wilcoxon signed-rank test, one-tailed, $p=0.001$, Fig. S1). Neurovascular activity and ChrimsonR expression were distributed similarly along the AP axis and their amplitudes were correlated (Fig. 1d, Fig. S1). Again, our control experiments demonstrated that direct optogenetic stimulation of a non-transfected cortical 


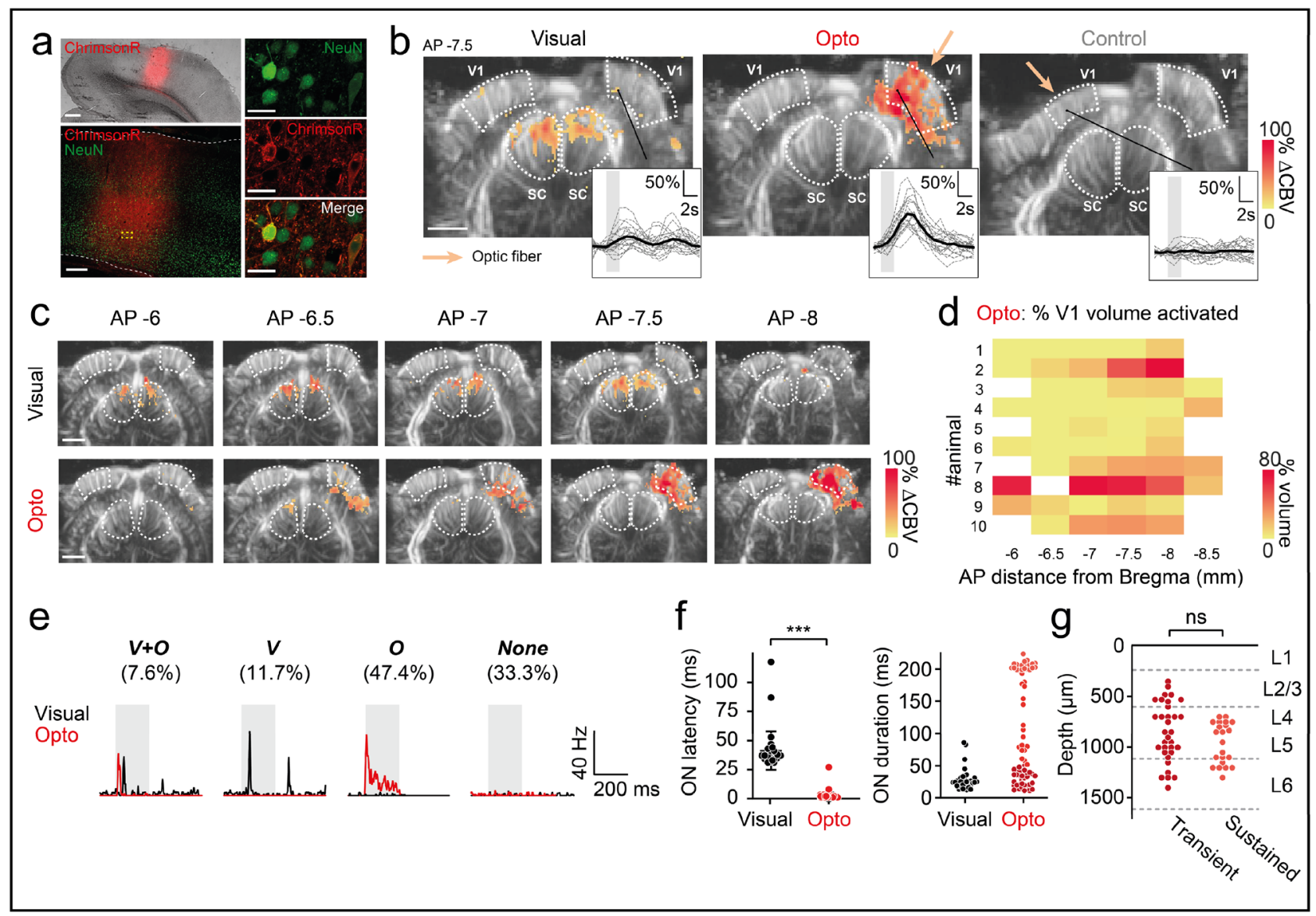

Figure 1. V1 neurovascular and neuronal activations resulting from cortical surface stimulation in rats. (a) Left: $2.5 \times$ imaging (top) and $20 \times$ confocal imaging of a brain section showing the localization of ChrimsonR in V1. White dashed lines delimit the cortical surface and border between V1 and the white matter. Right: close-up $(40 \times)$ of the area delimited by the yellow lines in the previous image, showing two ChrimsonR-expressing neurons. Scale bars: $500 \mu \mathrm{m}, 200 \mu \mathrm{m}, 20 \mu \mathrm{m}$. (b) fUS activation maps obtained after visual stimulation of the contralateral eye (left), optogenetic stimulation of the ipsilateral V1 area expressing ChrimsonR (middle) and control optogenetic stimulation of the uninjected contralateral V1 area (right), from a single imaging plane (AP $-7.5 \mathrm{~mm}$ ) from the same animal. White dashed lines delimit the V1 and SC areas. Colored pixels indicate significant CBV variation (Wilcoxon signed-rank test with Bonferroni-Holm correction). Right insets: patterns of single-pixel activation. Gray lines represent single-trial activity $(n=20)$ and the black line represents the mean CBV variation. Colored patches indicate light stimulation (duration: $2 \mathrm{~s}$ ) (c) fUS activation maps for visual and optogenetic activation, for all imaging planes (AP $-6 \mathrm{~mm}$ to $\mathrm{AP}-8 \mathrm{~mm}$ ) in which V1 (delimited by white dashed lines) is present, from the same animal (animal \#2). (d) Left: percentage of V1 activated during optogenetic stimulation for each fUS imaging plane, for all animals $(n=10)$. Right: AP distribution of the ChrimsonR expression area on brain sections. (e) Spike density function (SDF) of typical V1 single units during visual (black lines) or optogenetic (red lines) activation. Four subpopulations of neurons were identified: double-responsive $\left(\mathrm{n}_{\mathrm{V}+\mathrm{O}}=13\right)$, responsive to the visual $\left(\mathrm{n}_{\mathrm{V}}=20\right)$ or optogenetic stimulus only $\left(\mathrm{n}_{\mathrm{O}}=81\right)$, nonresponsive $\left(\mathrm{n}_{\mathrm{None}}=57\right)$. (f) Left: $\mathrm{ON}$ response latencies for visual ( $n=33$ units) and optogenetic ( $n=76$ units) stimulation (Mann-Whitney, $p<0.0001$ ). Right: ON response durations for visual ( $n=33$ units) and optogenetic stimulation ( $n=72$ units). (g) Depth profile of transient (ON duration $<51 \mathrm{~ms}, n=33$ units) and sustained (ON duration $>197 \mathrm{~ms}, n=22$ units) neurons activated by optogenetics. (c-d) Scale bar: $2 \mathrm{~mm}$. The irradiance used for optogenetic and control stimulation was $140 \mathrm{~mW} \mathrm{~mm}^{-2}$.

area resulted in no activation. The findings for these control conditions indicate that the parameters we used for optogenetic stimulation at the cortical surface did not generate CBV variations in areas into which the virus was not injected (mean: $0 \pm 0 \%$ ). These observations indicate that the optogenetic light stimulation used here does not trigger a vascular response detectable by fUS imaging. Consequently, with fUS imaging, we were able to visualize the entire volume of an optogenetically evoked response resulting from focal stimulation within the primary visual cortex.

We then sought to confirm that the observed changes in blood volume following optogenetic stimulation at the surface of V1 were indeed due to an increase in neuronal activity, and not to indirect factors, such as heating. We therefore performed electrophysiological recordings of V1 during the stimulation of the contralateral eye with white light $\left(200 \mathrm{~ms}, 1 \mathrm{~Hz}, 100\right.$ cycles, $\left.58 \mathrm{~mW} \mathrm{~cm}^{-2}\right)$ or of the injected V1 area with light at $595 \mathrm{~nm}(200 \mathrm{~ms}, 1 \mathrm{~Hz}$, 


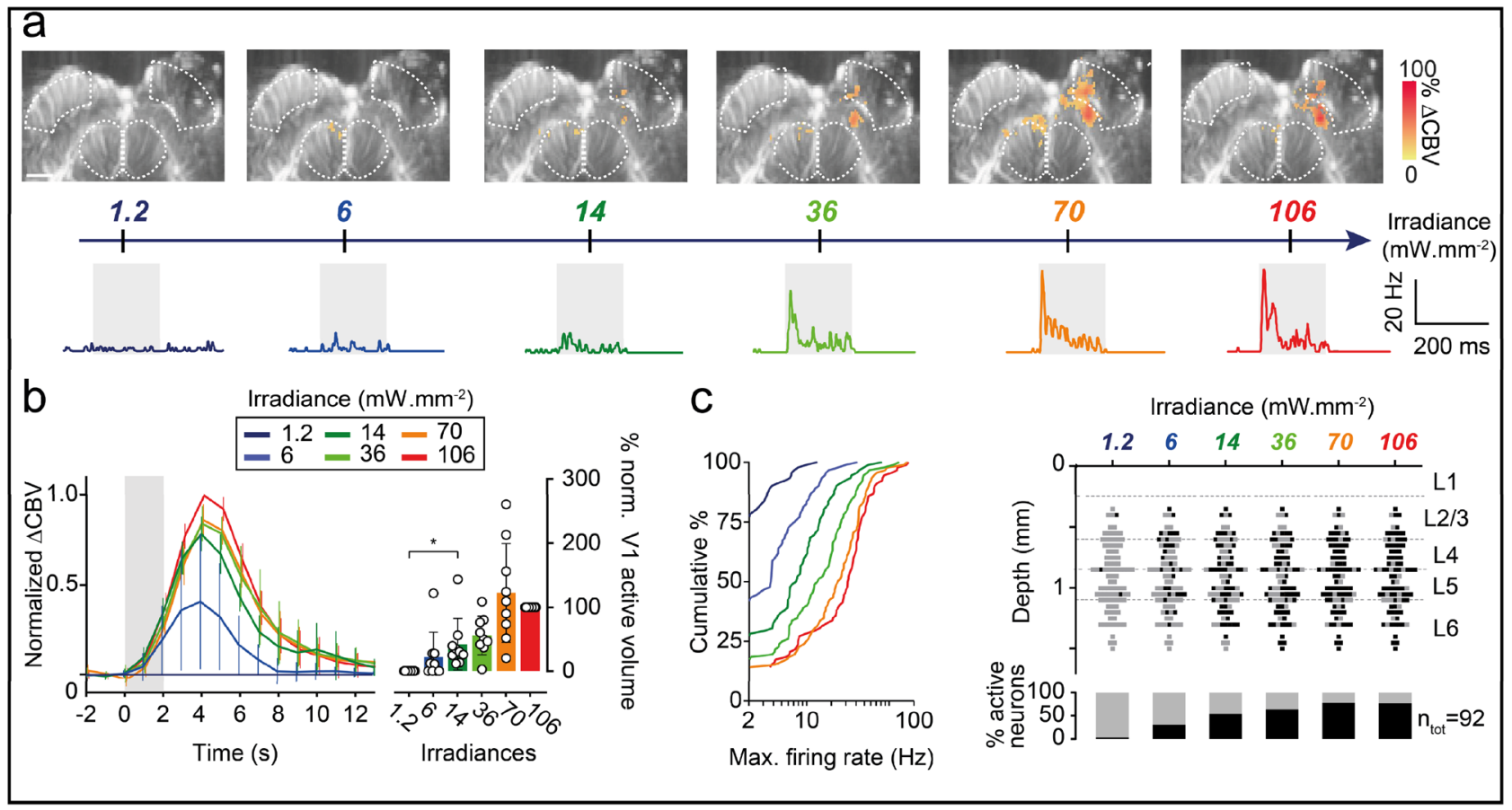

Figure 2. Dose-response relationship of optogenetic activations (a) Top: fUS activation maps of a single imaging plane (AP $-7.5 \mathrm{~mm}$ ) from animal \#4 at different irradiances. Scale bar: $2 \mathrm{~mm}$. White dashed lines delimit the V1 and SC areas. Bottom: SDF from a typical V1 single unit during optogenetic stimulation for $200 \mathrm{~ms}$ (gray patch) at different irradiances. (b) Left panel: mean CBV variation over all animals $(n=9)$ for each irradiance. of the values for each animal were normalized against those obtained at $106 \mathrm{~mW} \mathrm{~mm} \mathrm{~m}^{-2}$. Error bars represent the standard deviation. The colored patch corresponds to the $2 \mathrm{~s}$ optogenetic stimulation. Right panel: normalized active volumes of the ipsilateral V1 area for each irradiance. Open circles are individual values, bars represent the mean and the standard deviation ( $n=9$ animals, Wilcoxon signed-rank test, one-tailed, $p<0.05)$. (c) Left: cumulative distribution of the mean maximal firing rates of all recorded V1 single units $(n=92)$ during optogenetic stimulation, for each irradiance. Right panel, top: depth profile of V1 single units activated (black squares) or not (gray squares) by the optogenetic stimulation, for each irradiance ( $n=92$ single units). Bottom: percentage of active (black) and non-responsive (gray) neurons for each set of conditions.

100 cycles, $140 \mathrm{~mW} \mathrm{~mm}^{-2}$ ). We used the Spyking Circus algorithm ${ }^{34}$ to sort the multi-unit recordings, to obtain single-cell responses. In total, we recorded a population of 171 neurons from nine animals expressing ChrimsonR in V1. These neurons displayed several distinctive patterns of activity under both direct eye and optogenetic stimulation conditions (Fig. 1e). We plotted the spike density function (SDF) of four V1 neurons for both direct eye stimulation (black lines) and optogenetic conditions (red lines), to highlight the diversity of these activity patterns. Based on the profiles of visual and optogenetic responses, we classified neurons into four different groups: neurons responding to both visual and optogenetic stimulation $(\mathrm{V}+\mathrm{O}$ neurons, $n=13,7.6 \%$, Wilcoxon signed-rank test, two-tailed, $p<0.01$, between baseline: $[-1000] \mathrm{ms}$, and stimulus presentation window: $[0200]$ $\mathrm{ms}$ ), to visual stimulation only (V neurons, $n=20,11.7 \%$ ), to optogenetic stimulation only (O neurons, $n=81$, $47.4 \%$ ), and non-responsive neurons ('None' neurons, $n=57,33.3 \%$ ). Most of the neurons responding to visual stimulation displayed phasic responses, with an ON response occurring after the start of stimulation followed, in some cases, by an OFF response. By contrast, the neurons responding to optogenetic stimulation displayed a unique $\mathrm{ON}$ response. We characterized the neuronal activation further, by comparing the onset latencies and durations of the $\mathrm{V} 1$ responses for both direct eye and optogenetic stimulation conditions, for $\mathrm{V}, \mathrm{O}$ and $\mathrm{V}+\mathrm{O}$ neurons ( $n=109$, Fig. 1f). As observed for the representative neurons shown in Fig. 2a, the onset latencies of V1 responses were significantly shorter after optogenetic stimulation (mean: $1.76 \pm 3.14 \mathrm{~ms}, n=76$ units) than after stimulation of the contralateral eye (mean: $41.24 \pm 16.61 \mathrm{~ms}, n=33$ units, Mann-Whitney, $p<0.0001$ ). These very short response latencies for optogenetic stimulation are consistent with the direct activation of transfected neuronal cell bodies, bypassing all retinal synapses, by contrast to natural visual signal transmission. We also analyzed the duration of visual/optogenetic responses, to determine whether V1 neurons presented transient or sustained activity, according to the type of stimulation. For direct eye stimulation, the duration of neuronal responses was tuned on a single ensemble, with a mean duration of activation of $28.09 \pm 17.05 \mathrm{~ms}(n=33)$. By contrast, in optogenetic conditions, two subgroups emerged: neurons displaying transient and sustained responses. Based on these results, we defined two subsets of neurons: transient (durations $<51 \mathrm{~ms}, n=33$ ) and sustained (durations $>197 \mathrm{~ms}, n=22)$ neurons. The remaining neurons $(n=17)$ had intermediate response durations. The transient responses may result from inhibitory cortical feedback from interneurons or a difference in voltagegated channel properties between neuronal subtypes leading to the silencing of these neurons. We found no 


\begin{tabular}{|l|l|}
\hline Irradiance $\left({\left.\mathbf{m W ~} \mathbf{~ m m}^{-\mathbf{2}}\right)}^{\mathbf{2}}\right.$ & Power $(\mathbf{m W})$ \\
\hline 142 & 7.1 \\
\hline 140 & 7.0 \\
\hline 106 & 5.3 \\
\hline 70 & 3.5 \\
\hline 36 & 1.8 \\
\hline 14 & 0.7 \\
\hline 6 & 0.3 \\
\hline 1.2 & 0.06 \\
\hline
\end{tabular}

Table 1. $595 \mathrm{~nm}$ light irradiances and powers used in this study.

significant difference in the depth distribution of transient and sustained neurons (Fig. 1g), revealing an absence of correlation between neuronal response patterns and potential decreases in stimulus intensity with tissue depth; transient and sustained neurons were recorded within the same cortical layers (L2/3 to L6), suggesting a direct activation of cortical neurons by optogenetic stimulation at the cortical surface. We then assessed the specificity of optogenetic activation, by performing electrophysiological recordings on naive animals. In the animals in which no injection was performed (Fig. S1, $n=3$ animals), almost all the recorded neurons $(n=187 / 188)$ displayed a total absence of response to optogenetic stimulation; the only responsive neuron had a very low response rate $(3.89 \mathrm{~Hz})$ relative to its baseline activity $(1.71 \mathrm{~Hz}, p=0.0014)$. In this experiment, most neurons displayed a visual response when the contralateral eye was stimulated $(n=80 / 118)$, whereas another group of neurons $(n=37 / 118)$ did not respond to either visual or optogenetic stimulation. Thus, by recording single-cell activities in transfected areas of V1, we were able to demonstrate that optogenetic light stimulation at the surface of the cortex triggered both an increase in cerebral blood volume, as shown by fUS imaging, and direct neuronal activation. We can therefore conclude that the fUS variations we observed reflected the optogenetic activation of V1 neurons. As in fUS imaging, we found that a larger number of neurons responded to optogenetic stimulation than to visual stimulation (Fig. 1e), consistent with the broader activation of areas in response to optogenetic stimulation than following direct contralateral eye stimulation and fUS imaging.

fUS imaging and electrophysiological recordings reveal the dose-response relationship of optogenetic activations to different irradiances. As both single-cell recording and fUS imaging can be used to monitor optogenetic neuronal activation, we compared the responses obtained with these two techniques for different irradiances (for light power equivalences, see Table 1). We first imaged the same single V1 plane (AP $-7.5 \mathrm{~mm}$ ) by fUS while optogenetically stimulating the surface of the ChrimsonR-expressing V1 area with various irradiances (from 1.2 to $106 \mathrm{~mW} \mathrm{~mm}^{-2}$ ). The size of the active volume within the injected $\mathrm{V} 1$ area increased with irradiance, with a major increase between 36 and $70 \mathrm{~mW} \mathrm{~mm}^{-2}$ and a slight decrease at $106 \mathrm{~mW} \mathrm{~mm}^{-2}$ (Fig. 2a). One also found that the contralateral SC was slightly activated at irradiances above 1.2 $\mathrm{mW} \mathrm{mm} \mathrm{mm}^{-2}$. For each irradiance, we then calculated the mean CBV variation over all significant pixels in the V1 area and over all trials (Fig. 2b, left panel). For each animal, we normalized these values against those obtained for the highest irradiance $\left(106 \mathrm{~mW} \mathrm{~mm}^{-2}\right.$. Normalized CBV variation peaked $4 \mathrm{~s}$ after the start of stimulation. Peak CBV values increased as a function of irradiance. No CBV variation was recorded for the lowest irradiance $\left(1.2 \mathrm{~mW} \mathrm{~mm}^{-2}\right)$. Difference in peak CBV values relative to that for the lowest irradiance tested started to become significant from $6 \mathrm{~mW} \mathrm{~mm}^{-2}$ onwards $\left(1.2 \mathrm{~mW} \mathrm{~mm}^{-2}\right.$, mean: $0 \pm 0 ; 6 \mathrm{~mW} \mathrm{~mm}{ }^{-2}$, mean: $0.36 \pm 0.34$, Wilcoxon signed-rank test, one-tailed, $p<0.05)$. For each irradiance, we then calculated the percentage normalized activated ipsilateral V1 volume (Fig. $2 \mathrm{~b}$, right panel) in all animals $(n=9)$. We again observed an increase in the normalized active volume of V1 with irradiance. Difference with respect the value obtained at the lowest irradiance became significant from $6 \mathrm{~mW} \mathrm{~mm}^{-2}$ onwards $\left(1.2 \mathrm{~mW} \mathrm{~mm}^{-2}\right.$, mean: $0 \pm 0 \% ; 6 \mathrm{~mW} \mathrm{~mm}^{-2}$, mean: $22.3 \pm 39.0 \%$, Wilcoxon signed-rank test, one-tailed, $p<0.05$ ). We then investigated whether the variation of CBV responses observed with irradiance was related to the number of neurons recruited. The SDF from an example single-unit was determined for the different irradiances; the amplitude of this single-unit increased with irradiance: over 100 trials, this neuron had an average peak response of $8.2 \mathrm{~Hz}(\mathrm{SEM} \pm 3.87)$ for $6 \mathrm{~mW} \mathrm{~mm}^{-2}$ and $36.6 \mathrm{~Hz}(\mathrm{SEM} \pm 7.3)$ for $106 \mathrm{~mW} \mathrm{~mm}^{-2}$ (Fig. 2a). We generalized this analysis by plotting the cumulative distribution of the mean maximal firing rates for each irradiance (Fig. 2c, left panel). The cumulative curves reached a plateau for lower maximal firing rates with decreasing irradiance. We noted a significant difference in distribution between 1.2 $\mathrm{mW} \mathrm{mm} \mathrm{m}^{-2}$ and irradiances of $6 \mathrm{~mW} \mathrm{~mm}^{-2}$ and above $\left(1.2 \mathrm{~mW} \mathrm{~mm}^{-2}\right.$, median: $0 \mathrm{~Hz} ; 6 \mathrm{~mW} \mathrm{~mm} \mathrm{~mm}^{-2}, 3.8 \mathrm{~Hz}, \mathrm{Kol}-$ mogorov-Smirnov test, $p<0.0001)$, demonstrating that electrophysiological recordings and fUS imaging had equivalent sensitivities. Finally, in our total population of V1 neurons, the proportion of responsive neurons increased with irradiance (from $28 / 92$ units at $6 \mathrm{~mW} \mathrm{~mm}^{-2}$ to $71 / 92$ units at $106 \mathrm{~mW} \mathrm{~mm}^{-2}$, Fig. $2 \mathrm{c}$, right panel). Interestingly, the depth distribution of the activated neurons did not change with increasing irradiance (Fig. 2c, right panel). It was, therefore, possible to activate neurons from deep cortical layers even at very low irradiances.

Spread of optogenetic activation to downstream and upstream visual areas. The results described above relate to optogenetic activation in V1 with the optic fiber placed at the surface of the primary visual cortex. We then investigated whether the activity initiated in V1 was functionally relevant and spread to 


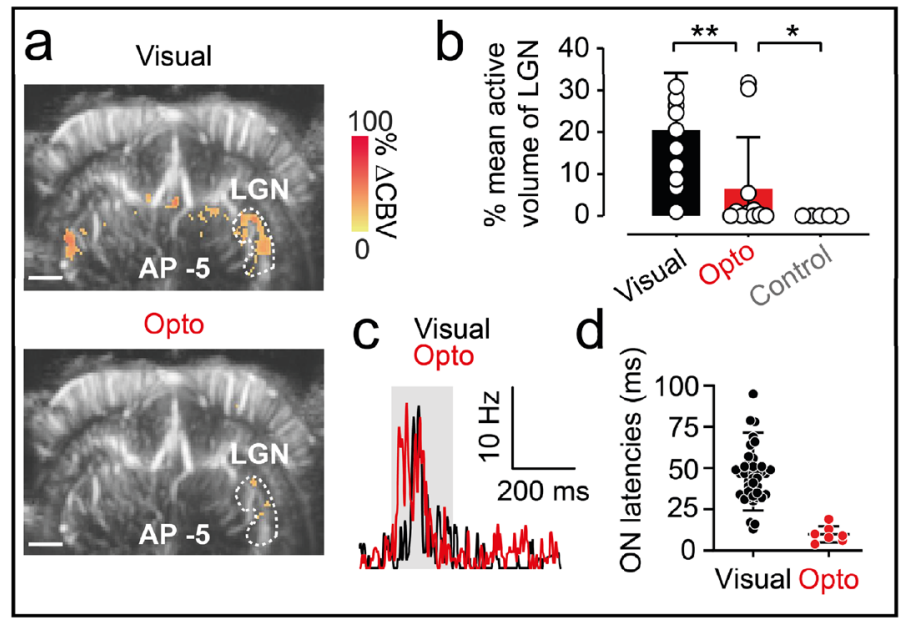

Figure 3. Spread of optogenetic activation to the LGN. (a) fUS activation maps obtained during visual stimulation of the contralateral eye (top) and optogenetic stimulation of the ChrimsonR-expressing ipsilateral $\mathrm{V} 1$ area (bottom) from a single imaging plane in which the LGN is present (AP $-5 \mathrm{~mm}$ ) from the same animal. White dashed lines delimit the ipsilateral LGN. Scale bar: $2 \mathrm{~mm}$. (b) Percentage active volume of the LGN after visual, optogenetic or control stimulation. For both visual and optogenetic stimulations, we show the volumes of the LGN ipsilateral to the injection, whereas, for control stimulations, the volume of the contralateral LGN is shown. Open circles represent mean values over all imaging planes for each animal (visual and optogenetic stimulation, $n=11$, Wilcoxon signed-rank test, one-tailed, $p=0.0068, n=5$ for control, Mann-Whitney test, one-tailed, $p=0.0288$ ), bars represent the mean for all animals. (c) SDF of a typical LGN single unit responding to visual (black line) and optogenetic (red line) stimulation of the ipsilateral V1 area. (d) ON latencies of V1 and LGN single-unit responses to visual (V1, $n=33$ units, LGN, $n=42$ units) or optogenetic stimulation of V1 (V1, $n=29$ units, LGN, $n=7$ units. Mann-Whitney test, $p<0.05)$. The irradiance used for optogenetic and control stimulation was $142 \mathrm{~mW} \mathrm{~mm}^{-2}$.

other visual structures up- or downstream. For the downstream pathway, LGN terminals end in V1 at the depth of cortical layer IV, whereas cortical V1 layer VI sends feedback connections to the LGN. We thus hypothesized that injecting AAV9-7m8-CaMKII-ChrimsonR-tdT into V1 would increase ChrimsonR expression in at least one of these two categories of fibers and that the optogenetic activation of V1 would lead to direct activation of the LGN via LGN terminals in V1, or to an indirect activation of the LGN through feedback connections in V1 cortical layer VI. Histological analyses confirmed that some ChrimsonR expression occurred in the LGN, but we were unable to identify any ChrimsonR-expressing cell bodies in the LGN, suggesting that only retrograde fibers originating from cortical V1 layer VI expressed this opsin (Fig. S2). We imaged the planes containing the LGN (AP -3.5 to AP $-5.5 \mathrm{~mm}$ ). Figure $3 \mathrm{a}$ shows the fUS imaging planes at AP $-5 \mathrm{~mm}$ for the direct eye and optogenetic stimulation conditions. We noted a slight activation of the visual cortex following visual stimulation (see Fig. 1b), and a strong activation of the ipsilateral LGN ( $n=85 / 225$ active pixels) when the contralateral eye was stimulated with the white LED, suggesting that this kind of stimulation was more appropriate for LGN and SC activations than for the visual cortex. Indeed, in the 11 animals (Fig. 3b), the mean percentage of the LGN volume visually activated was $20.5 \pm 13.7 \%$ which is much greater than the volume activation obtained for V1 (less than 1\%). When the injected V1 surface was stimulated with the optic fiber (Fig. 3a), CBV responses increased significantly in the ipsilateral LGN, but with a much smaller number of activated pixels $(n=12 / 225$ pixels) than for visual stimulation. We also performed a control optogenetic stimulation, in which we stimulated the non-injected V1 area. We observed no activation of the ipsilateral LGN, confirming that, on stimulation of the injected hemisphere, ipsilateral LGN activation was due to optogenetic activation of LGN terminals in V1 or feedback from the V1 area (Fig. S2). In the 11 animals tested, the active LGN volumes for visual stimulation were larger than those for optogenetic stimulation (visual, mean: $20.5 \pm 13.7 \%$; optogenetic, mean: $6.5 \pm 12.3 \%$, Wilcoxon signed-rank test, $p=0.0068$ ). In addition, for the five animals tested by optogenetic stimulation of the non-transfected V1 area, we detected no significant responsive pixels (Fig. $3 \mathrm{~b}$, mean: $0 \pm 0 \%, n=5$ animals). We also performed single-cell recordings in the LGN, to demonstrate that the neurovascular activations imaged by fUS in the LGN coincided with the activation of LGN neurons (Fig. 3c). We recorded a total of 153 neurons in the LGN. Only two units responded to both visual and optogenetic stimulation; seven units were responsive only to optogenetic stimulation, and 40 units were responsive only to visual stimulation ( $n=7$ animals). The vast majority of LGN neurons were unresponsive to both types of stimulation. The distribution of onset latencies following direct eye stimulation was broader for LGN than for V1 single units (Fig. 3d, mean: $47.88 \pm 23.55 \mathrm{~ms}$, $n=42$ units, and see Fig. 1f). This may reflect the recording of both cells activated by retinal ganglion cells and cells retrogradely activated by the V1 area, which have higher latencies. Following optogenetic stimulation at the surface of the V1 area, the onset latencies for LGN neurons were shorter than those following visual stimulation (mean: $9.86 \pm 4.95 \mathrm{~ms}, n=7$ units, Mann-Whitney, $p<0.05$ ). However, these response latencies were greater than those recorded in V1 ( $n=42$ units. Mann-Whitney, $p<0.0001$, see Fig. 1f for V1 units). This result suggests that 


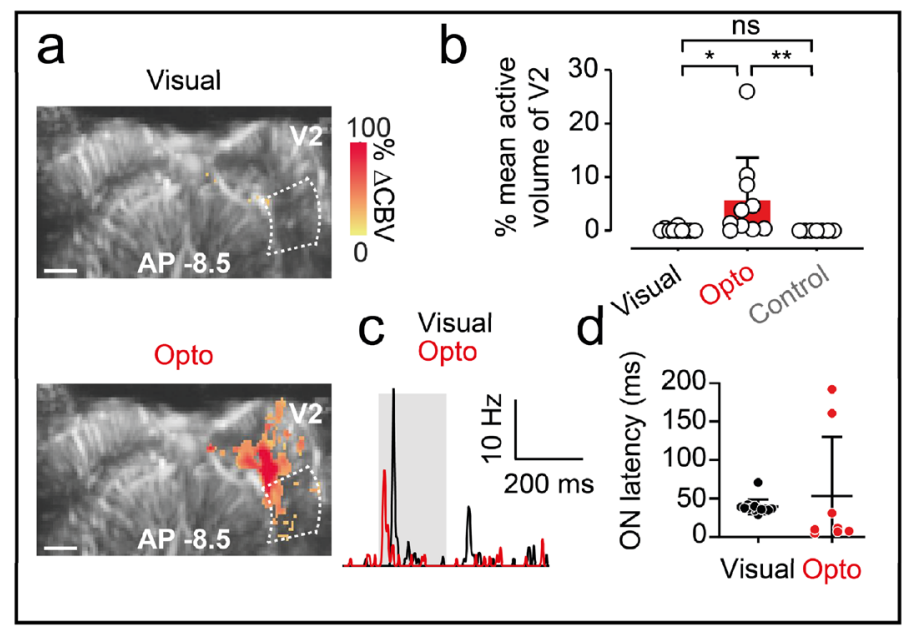

Figure 4. Spread of the optogenetic activation to V2. (a) fUS activation maps obtained during visual stimulation of the contralateral eye (top) and optogenetic stimulation of the ChrimsonR-expressing ipsilateral $\mathrm{V} 1$ area (bottom) and control stimulation of the uninjected contralateral area from a single imaging plane (AP $-7.5 \mathrm{~mm}$ ) containing the V2 area. White dashed lines delimit the ipsilateral V2 area. Scale bar: $2 \mathrm{~mm}$. (b) Percentage active volume of V2 after visual, optogenetic or control stimulation. For both visual and optogenetic stimulation, we show the volumes of V2 ipsilateral to the injection, whereas, for the control, we shown the volume of the contralateral V2. Open circles represent mean values over all imaging planes for each animal (visual and optogenetic, $n=1$, Wilcoxon signed-rank test, one-tailed, $p=0.002, n=7$ for control, Mann-Whitney test, one-tailed, $p=0.0004$ ), bars represent the mean over all animals. (c) SDF of a typical V2 single unit responding to visual (black line) and optogenetic (red line) stimulation of the ipsilateral V1 area. (d) ON latencies of V1 and V2 single-unit responses to visual (V1, $n=33$ units, V2, $n=14$ units) or optogenetic stimulation of $\mathrm{V} 1$ (V1, $n=76$ units, $\mathrm{V} 2, n=8$ units). The irradiance used for optogenetic and control stimulation was $140 \mathrm{~mW} \mathrm{~mm}^{-2}$.

the optogenetically activated LGN single units recorded here were activated indirectly, by retrograde fibers from V1 cortical layer VI, as suggested by the histological data.

We also investigated whether V1 optogenetic stimulation could spread to the direct upstream visual area toward which V1 projects: the V2 area. We obtained fUS activation maps in different imaging planes (AP -6 to $\mathrm{AP}-8 \mathrm{~mm}$ ) in which the entire V2 area was present after either direct stimulation of the contralateral eye or optogenetic stimulation at the surface of the V1 area. We show representative activation maps from single imaging planes (AP $-8.5 \mathrm{~mm}$ ) in Fig. 4a. Visual stimulation of the contralateral eye led to almost no activation of the V2 area on the same side as the injected V1. By contrast, optogenetic stimulation of the transfected $\mathrm{V} 1$ area led to a stronger activation of the ipsilateral V2, mostly within the ventral part of V2. As previously reported, within the different imaging planes, we also observed strong activation in the lower parts of V1 and $\mathrm{V} 2$ containing the axons. We next quantified the active volume over all imaging planes for the V2 area, for all 10 animals (Fig. 4b). As for the V1 area, visual stimulation resulted in only weak activation of V2 (mean: $0.2 \pm 0.3 \%$ of activated volume). Averaged activation volumes were significantly larger for optogenetic stimulation than for visual stimulation (mean: $5.6 \pm 8.0 \%$ activated volume, $n=10$ animals, Wilcoxon signed-rank test, one-tailed, $p<0.05)$. Control stimulation of the non-injected V1 area confirmed the specificity of the spread of optogenetic activation from V1 to V2, as no activation was observed in the contralateral V2 area (Fig. 4b, Fig. S2).

Electrophysiological recordings within V2 confirmed that the fUS variations we observed in V2 were consistent with direct neuronal activation (Fig. 4c). We recorded V2 single units responding to visual and optogenetic stimulation. As previously described, we characterized the onset latency of these neurons, comparing the values obtained with those for V1 single units (Fig. 4d). Similar visual latencies were recorded for V1 and V2 single units (visual stimulation: V1, mean: $41.24 \pm 16.61 \mathrm{~ms}, n=33$ units (see Fig. 1f); visual stimulation: V2, mean: $39.43 \pm 9.557 \mathrm{~ms}, n=14$ units). Interestingly, optogenetic onset latencies were higher for $\mathrm{V} 2$ single units than for V1 (V1, mean: $1.76 \pm 3.14 \mathrm{~ms}, n=76$ units. V2, mean: $62.78 \pm 77.44 \mathrm{~ms}, n=9$ units, Mann-Whitney, $p<0.0001$ ), suggesting that these neurons were activated indirectly by ChrimsonR-expressing V1 neurons. Two V2 neurons presented particularly long optogenetic ON latencies, possibly due to a difference in the microcircuits involved. We checked that this variability did not bias the latency delay for V2 and V1 neurons after optogenetic stimulation, by performing the same statistical test on a V2 data set restricted to the remaining seven fast V2 units. The difference in ON latency between V1 neurons and these fast V2 neurons was conserved (fast V2 units, mean: $12.2 \pm 9.5 \mathrm{~ms}, n=7$ units, Mann-Whitney, $p<0.0001$ ), suggesting that all V2 neurons were activated indirectly after the onset of stimulation. This conclusion is supported by the lack of ChrimsonR expression in V2 on brain slices from the animals used to record these single units. However, these V2 latencies were shorter than V2 latencies for natural eye stimulation, demonstrating that they were genuinely produced by V1 optogenetic activation. 


\section{Discussion}

Coupling of optogenetics with fUS. In this study, we demonstrate that optogenetic activation can be detected by fUS imaging. Rungta and colleagues ${ }^{25}$ indicated that blue light from the tip of the optic fiber per se could induce neurovascular responses in naïve mice, at light powers higher than $2 \mathrm{~mW}$ (irradiances higher than $18 \mathrm{~mW} \mathrm{~mm}^{-2}$ ). Two other recent studies reported hemodynamic responses following blue light illumination of the retrosplenial cortex in Thy-ChR2 mice or the illumination of specific neuronal subpopulations of the superior colliculus in other transgenic lines ${ }^{19,26}$. We here show that even with AAV-mediated expression of microbial opsins, we can specifically activate neurons by optogenetics but in the red range which provides deeper light penetration and less light scattering. The authors explained that they used a lower light power and irradiance $\left(0.3 \mathrm{~mW} / 1.5 \mathrm{~mW} \mathrm{~mm}^{-2}\right)$ to prevent non-specific activation. By contrast, we used an AAV-mediated strategy to express ChrimsonR in V1 neurons, resulting in a lower density of opsin-expressing cells than in transgenic mice lines ${ }^{35}$. Our results demonstrate the possibility of recording neurovascular optogenetic activation due to a small number of transfected neurons. Moreover, we show here that stimulation of the control non-injected hemisphere does not induce a vascular response, thereby demonstrating the specificity of ChrimsonR-elicited optogenetic activity. Single-cell recordings confirmed the neuronal optogenetic activation correlated with these CBV variations. We also used a higher wavelength $(595 \mathrm{~nm})$ for illumination than Rungta and coworkers, but with comparable values of power and irradiance. These results indicate that red light can be used even at high power, in protocols combining the optogenetic activation of red-shifted opsins and fUS imaging. Importantly, electrophysiological recordings from the control animals showed no neuronal activation, suggesting negligible thermal effects with the use of $595 \mathrm{~nm}$ light under the parameters used here. Indeed, we included these parameters in the heat propagation model of Stujenske ${ }^{36}$, and recorded a very local $(<1 \mathrm{~mm}$-diameter sphere from the tip of the optic fiber) increase in temperature, by $0.2^{\circ} \mathrm{C}$, which is too small to alter neuronal firing rates. Finally, we characterized the dose-response dependence of our recorded optogenetic activations.

The use of fUS imaging to analyze optogenetic responses has the advantage that it could potentially provide a mesoscopic view of the activated area. Unlike electrophysiological recordings, which extract information very locally $^{6}$, and calcium imaging, which is dependent on both expression of the calcium indicator and the penetration of blue light through the tissue ${ }^{37}$, it can display information at a brain-wide spatial scale. The coupling of fMRI with optogenetics meets these criteria, but with a lower spatial resolution ${ }^{8,10,11}$ (of the order of a millimeter per pixel). By contrast, fUS imaging can detect brain activity at a submesoscopic resolution $\left(100 \times 100 \mu \mathrm{m}^{2}\right)$, with less cumbersome equipment than for fMRI.

Spread of the activity within V1. We found that optogenetic neurovascular activation was well correlated with ChrimsonR expression in the AP axis. We also noted that optogenetic V1 stimulation spread beyond the borders of V1, as indicated by the activation of both the LGN and V2 on both fUS and electrophysiology. This spread of activity may be due to fibers located at the base of V1, connected to the LGN and expressing ChrimsonR (Fig. S1). These fibers may generate the neurovascular activity detected ventrally to V1 on our activation maps. Furthermore, the mean neuronal transfection rate was $5.5 \%$ in the coronal plane displaying the highest fluorescence signal, whereas a larger volume of V1 was activated in this plane. A first explanation for this may be the spread of neuronal activation to other V1 cells, although we recorded a rather homogeneous set of short-onset latencies. Alternatively, neurovascular activations may be broader per se than neuronal responses. Some studies of rat olfactory bulb glomeruli have provided evidence of a close overlap between capillary blood flow and neuronal activity ${ }^{38}$, whereas others have reported a mismatch between the areas of neurovascular and neuronal activation ${ }^{39,40}$. Specifically for V1, a lack of correlation between BOLD signals and spiking activity has been observed in cats ${ }^{41}$, and a lack of correlation between single-vessel hemodynamic responses and calcium imaging signals has been found in cats and rats $^{42}$.

Cortical visual restoration. We detected neurovascular and neuronal responses, even at low irradiance (6 $\mathrm{mW} \mathrm{mm} \mathrm{m}^{-2}$ ), with no modification of the depth distribution of the activated neurons. One recent study ${ }^{43}$ reported that the stimulation of deep V1 layers $(>1.5 \mathrm{~mm})$ in non-human primates with electrical prostheses elicited behavioral responses. Our ability to activate neurons in deep cortical layers highlights the potential value of redshifted opsin ChrimsonR for optogenetic cortical vision restoration strategies. Although it would still require a cranial surgery for the implantation of an optic device above the dura, the optogenetic therapy would bypass the need to insert electrodes in the tissue unlike electrical cortical visual restoration. Besides, long-term insertion of electrodes in the cortex leads to signal degradation over time, an issue that an optogenetic strategy can bypass too. A key element of visual restoration is the induction of neuronal responses with characteristics matching those resulting from a natural visual stimulus ${ }^{44-50}$. We show here that the firing rates induced by optogenetic stimulation were similar to those induced by visual stimulation. Optogenetically activated neurons had very short latencies, of the order of 1-2 ms. Theoretically, this is sufficient for the encoding of natural images into optogenetic stimulations at a temporal resolution matching the resolution of the natural visual signal. Moreover, those optogenetic onset latencies were quite homogeneous, despite being obtained for neurons located in different layers. These results demonstrate that we can induce a signal that does not lose its temporal resolution with depth.

Finally, the feasibility of inducing visual percepts by optogenetic cortical vision restoration remains to be demonstrated. In species with a more complex hierarchical organization of cortical visual areas, such as nonhuman primates, a few studies have shown behavioral effects due to the optogenetic stimulation of higher visual areas. Jazayeri ${ }^{51}$ and coworkers reported that saccades following fixation tasks were shifted towards the receptive field of the region of V1 optogenetically activated following the fixation point offset. Ju $\mathrm{u}^{52}$ and coworkers demonstrated the successful detection of optogenetic percepts in a saccade task. However, we used here a single 
optic fiber for optogenetic stimulation that led to a global activation of V1. This will hardly allow the encoding of precise spatial stimuli. The use of lower irradiances led to more restricted volumes of activations as shown in Fig. 2, which is more suitable for increasing the spatial resolution of the encoding. Subpopulation or single-cell activation can be achieved using more sophisticated optic techniques such as two-photon microscopy and holographic stimulation. However, coupling these activations techniques with fUS imaging remains challenging due to the hindrance of both an objective and a fUS probe onto a rodent cranial window. Moreover, concerning the optogenetic cortical therapy, the use of multiphoton microscopy will hardly be compatible with the development of easily-implantable prosthetics. Nevertheless, we showed a here a way to appraise the optogenetic activation volumes. Such information will be critical for later development of more complex stimulation system, such as arrays of micro-LEDs that will offer better spatial resolution. This might make it possible to generate percepts more complex than phosphenes and to develop discrimination behavioral tasks to assess their perception.

\section{Materials and methods}

The study was carried out in compliance with the ARRIVE guidelines.

Animals. All animal experiments and procedures were approved by the Local Animal Ethics Committee Charles Darwin CEEACD \#5 (registration number 2018032911282465) and performed in accordance with European Directive 2010/63/UE. We used wild-type male Long-Evans rats (Janvier Laboratories), nine weeks old at the time of viral injection. Rats were maintained under a reverse 12-h light/12-h dark cycle, with ad libitum access to food and water, except during surgery and electrophysiological recordings.

AAV production. The AAV9-7m8-CaMKII-ChrimsonR-tdT vector was packaged as previously described, by the triple transfection method, and purified by iodixanol gradient ultracentrifugation ${ }^{53}$. The AAV9$7 \mathrm{~m} 8$-CaMKII-ChrimsonR-tdT vector was titered by qPCR with SYBR Green ${ }^{54}$ (Thermo Fisher Scientific). The titer used in this study was $4.39 \times 10^{12} \mathrm{vg} \mathrm{\textrm {mL } ^ { - 1 }}$.

Immunostaining and confocal imaging. Following electrophysiological recordings, rats were euthanized, and their brains were extracted and fixed by overnight incubation in 4\% paraformaldehyde (100496, Sigma-Aldrich) at $4{ }^{\circ} \mathrm{C}$. Brains were then cryoprotected in $30 \%$ sucrose (84097, Sigma-Aldrich) and $50 \mu \mathrm{m}$ sagittal slices were cut with a microtome (HM450, Microm). The slices with the most intense tdT fluorescence from each brain were selected for further immunohistochemistry and imaging. Cryosections were permeabilized by incubation with $0.5 \%$ Triton X-100 in PBS for $1 \mathrm{~h}$ at room temperature and were then incubated in blocking buffer (PBS $+1 \% \mathrm{BSA}+0.1 \%$ Tween 20 ) for $1 \mathrm{~h}$ at room temperature. Samples were incubated overnight at $4{ }^{\circ} \mathrm{C}$ with monoclonal anti-NeuN antibody (1:500; Mouse, MAB377, Merck Millipore), in a 50\% dilution of blocking buffer $+0.5 \%$ Triton X-100. Secondary antibodies conjugated with Alexa Fluor dyes (1:500; Molecular Probes) and DAPI (1:1000, D9542, Merck Millipore), were incubated with the samples for $1 \mathrm{~h}$ at room temperature. An Olympus FV1000 laser-scanning confocal microscope with a 20× or 40× objective (UPLSAPO 20XO, NA: 0.85) was used to acquire images of brain sections.

Viral injections. Viral injections were performed in aseptic conditions with a digital small-animal stereotaxic instrument (David Kopf Instruments). Ear bars were covered with xylocaine to ensure that the animals felt no pain. Rats were anesthetized in a sealed box containing gaseous isoflurane (5\%), and maintained under anesthesia in the stereotaxic frame (25\% ketamine, $10 \%$ medetomidine and $65 \%$ saline injected intraperitoneally) for the entire surgical procedure, and animal body temperature was maintained at $37^{\circ} \mathrm{C}$ with a heating pad. Buprenorphine was injected subcutaneously to reduce inflammation, and Lubrithal was applied to the eyes to prevent them from drying out. Xylocaine, $70 \%$ ethanol and Vetedine were applied successively to the scalp before incision, to minimize pain and maintain sterile conditions. Cranial sutures were cleaned to remove connective tissue, by applying $\mathrm{H}_{2} \mathrm{O}_{2}$, to facilitate localization of the injection coordinates. We injected a total volume of $1.2 \mu \mathrm{L}$ of viral suspension unilaterally into rates, via two injection tracks, at a flow rate of 50-75 nL/ min, with a 5 or $10 \mu \mathrm{L}$ microsyringe (Hamilton) equipped with a microinjector (Sutter Instrument) and controller (World Precision Instruments). The coordinates for viral injection were $+2.8 /+3.2 \mathrm{~mm}$ from midline (M-L axis), $-6.5 /-7.5 \mathrm{~mm}$ from Bregma (A-P axis) and 1.6-1.35-1.1 mm ventral to the skull surface (D-V axis), based on the 2004 edition of the Paxinos and Franklin rat brain atlas. Viral efflux was prevented by leaving the needle in a $1.8 \mathrm{~mm}$ ventral position for two minutes before beginning the injection, with a three-minute interval left between injections before complete withdrawal of the needle from the cortex. After surgery, rats were brought round from anesthesia with a subcutaneous injection of Antisédan $(0.15 \mathrm{~mL})$.

In vivo electrophysiological recordings. Bilateral craniotomies were performed with a digital smallanimal stereotaxic instrument (David Kopf Instruments), at least 30 days after viral injection, to allow time for opsin expression. Ear bars were covered with xylocaine to prevent pain. Rats were anesthetized in a sealed box containing gaseous isoflurane (5\%) and maintained under anesthesia in the stereotaxic frame (25\% ketamine, $10 \%$ medetomidine and $65 \%$ saline injected intraperitoneally) for the entire surgical procedure, and electrophysiological recordings were taken with body temperature maintained at $37^{\circ} \mathrm{C}$ with a heating pad. Buprenorphine was injected subcutaneously to reduce inflammation, and Lubrithal was applied to the eyes to prevent them from drying out. Cranial sutures were cleaned to remove connective tissue, by applying $\mathrm{H}_{2} \mathrm{O}_{2}$, to reveal the injection tracks. Parietal bones were removed by drilling rectangular flaps and gently moving the bone away from the dura mater, exposing the cortex from 3 to $8.5 \mathrm{~mm}$ from Bregma (A-P axis), to cover the injection tracks. The dura was 
then gently removed. During drilling, the skull was regularly cooled with PBS, and once the cortex was exposed, it was protected from dehydration by the regular application of cortex buffer. After surgery, electrophysiological recordings were performed with 16-channel electrodes $(\mathrm{A} 1 \times 16-5 \mathrm{~mm}-50-703-O A 16 \mathrm{LP})$ coupled with a $400 \mu \mathrm{m}$-core fiber (Thorlabs M79L005 Fiber Cable, MM, $400 \mu \mathrm{m} 0.39 \mathrm{NA}, \mathrm{FC} / \mathrm{PC}$ to $1.25 \mathrm{~mm}$ ferrule, $0.5 \mathrm{~m}$ ) at various positions close to the injection sites. Electrophysiological data were acquired with MC RACK software. For visual stimulation, a white LED (Thorlabs MNWHL4 Mounted LED, $60 \mathrm{~mW} / \mathrm{cm}^{2}$ ) was placed $15 \mathrm{~cm}$ in front of the eye on the contralateral side of the cranial window. Electrophysiological recordings were digitized with a 16-channel amplifier (model ME32/16-FAI- $\mu \mathrm{PA}$, MultiChannel Systems). The sampling rate was $25 \mathrm{kHz}$.

Acquisition protocol for electrophysiological recordings. For optogenetic stimulation, we connected the optic fiber (reference above) to a light source (Thorlabs M595F2 (fiber coupled LED @595 nm), Ø400 $\left.\mu \mathrm{m}, 150 \mu \mathrm{W} / \mathrm{cm}^{2}\right)$ delivering light at the ChrimsonR excitation wavelength $(595 \mathrm{~nm})$. We targeted the transfected region of V1 by imaging tdT fluorescence with a Micron IV imaging microscope (Phoenix Research Laboratories) before recordings. The fiber was placed on the surface of the cortex while the electrode was inserted in the tissue. Both stimulations consisted of 100 repeats of $200 \mathrm{~ms}$ flashes at $1 \mathrm{~Hz}$. The onset of the flashes was aligned with electrophysiological data, with Clampex 9.2 software. We used several different irradiances of light at $595 \mathrm{~nm}$ in this study. Power at the fiber tip was measured with a power meter (Thorlabs, PM100D), by placing the fiber tip in contact with the sensor. The irradiance corresponding to each power was calculated as previously described $^{25}$.

Spike sorting. Offline spike sorting of the electrophysiological recordings (linear 16-channel electrodes) was performed with the SpyKING CIRCUS package ${ }^{34}$. Raw data were first high-pass filtered $(>300 \mathrm{~Hz})$ and spikes were detected when a filtered voltage trace crossed the threshold. Automatic cluster extraction was performed and candidate clusters were curated. Refractory period violations $(<2 \mathrm{~ms},>1 \%$ violation $)$ and noisy spike shapes led to cluster deletion. Spike templates with coordinated refractory periods in the cross-correlogram together with similar waveforms led to the merging of cluster pairs.

Electrophysiological data analysis. All electrophysiological data were extracted and analyzed with a custom-made Matlab script. Responsive units were defined as those displaying a significant difference in neuronal activity between the pre-stimulation period (averaged $100 \mathrm{~ms}$ before stimulus onset) and the stimulation interval (averaged $200 \mathrm{~ms}$ following stimulus onset, Wilcoxon signed-rank test, $p<0.01$ ). For each unit, responses are represented as the spike density function (SDF), which was calculated from the mean peristimulus time histogram (PSTH, bin size: $1 \mathrm{~ms}, 100$ trials) smoothed with a Gaussian filter (2 ms SD).

Calculation of latencies and response durations. The latency of the units displaying activation was defined as the first time point at which the SDF crossed the value of the baseline plus 2SD and remained higher than this value for at least $10 \mathrm{~ms}$. Conversely, the offset of activation was defined as the first time point after latency that the SDF crossed back below the value of the baseline plus 2SD and remained lower than this value for at least $10 \mathrm{~ms}$. Not all active neurons from a given population met these criteria, accounting for the slight difference between the number of active neurons and the number of latencies presented here.

Generation of functional ultrasound images. fUS imaging was performed as previously described ${ }^{17}$, with a linear ultrasound probe (128 elements, $15 \mathrm{MHz}, 110 \mu \mathrm{m}$ pitch and $8 \mathrm{~mm}$ elevation focus, Vermon; Tour, France) driven by an ultrafast ultrasound scanner (Aixplorer, Supersonic Imagine; Aix-en-Provence, France).

Acquisition protocol for fUS imaging. 3D fUS acquisitions were performed after craniotomy and electrophysiological recordings, as previously described. When optogenetic responses were observed, the position of the optic fiber on the surface of the cortex was kept unchanged until control acquisitions were performed, in which the fiber was moved to the other hemisphere. The cortex buffer on the surface of the cortex dried out, and $1 \mathrm{~cm}^{3}$ of ultrasound coupling gel was placed between the cortex and the linear ultrasound probe. Acquisition protocols consisted of 20 stimulation blocks, each consisting of $13 \mathrm{~s}$ of rest followed by $2 \mathrm{~s}$ of stimulation. For visual stimulation, the white LED used for electrophysiological recordings was kept in the same position, and the $2 \mathrm{~s}$ stimulation consisted of eight repeats of $125 \mathrm{~ms}$ flashes at $4 \mathrm{~Hz}$. For optogenetic stimulation, the $2 \mathrm{~s}$ stimulation consisted of 40 repeats of $25 \mathrm{~ms}$ flashes at $20 \mathrm{~Hz}$. Acquisitions were performed on coronal planes from 3 to $8.5 \mathrm{~mm}$ from Bregma (AP axis), with a $0.5 \mathrm{~mm}$ increment corresponding to the thickness of the imaging plane.

Building activation maps. For each pixel, we averaged, for each block, the intensity of Doppler power at baseline ( $2 \mathrm{~s}$ before stimulus onset) and during a response window (4 $\mathrm{s}$ after stimulus onset). The signals were then compared in one-tailed Wilcoxon signed-rank tests. Only pixels with $p$-values $<4.03 \times 10^{-6}$ (corresponding to a global $p$-value $<0.05$ with Bonferroni correction) were considered significant. On the maps, CBV during the response window is presented as a percentage of the baseline value. Region of interest (ROI) as V1, V2, SC and LGN were determined for each imaging plane, based on the Matt Gaidica rat brain atlas.

\section{Data availability}

The datasets generated during and/or analyzed during the current study are available from the corresponding author on reasonable request. 
Received: 12 May 2021; Accepted: 31 May 2021

Published online: 15 June 2021

\section{References}

1. Deisseroth, K. Optogenetics: 10 years of microbial opsins in neuroscience. Nat. Neurosci. 18, 1213-1225 (2015).

2. Carrillo-Reid, L., Yang, W., Bando, Y., Peterka, D. S. \& Yuste, R. Imprinting and recalling cortical ensembles. Science 353, 691-694 (2016).

3. Carrillo-Reid, L., Han, S., Yang, W., Akrouh, A. \& Yuste, R. Controlling visually guided behavior by holographic recalling of cortical ensembles. Cell 178, 447-457.e5 (2019).

4. Gill, J. V. et al. Precise holographic manipulation of olfactory circuits reveals coding features determining perceptual detection. Neuron 108, 382-393.e5 (2020).

5. Pégard, N. C. et al. Three-dimensional scanless holographic optogenetics with temporal focusing (3D-SHOT). Nat. Commun. 8, $1-14$ (2017)

6. Buzsáki, G., Anastassiou, C. A. \& Koch, C. The origin of extracellular fields and currents: EEG, ECoG LFP and spikes. Nat. Rev. Neurosci. 13, 407-420 (2012).

7. Lee, J. H. et al. Global and local fMRI signals driven by neurons defined optogenetically by type and wiring. Nature 465, 788-792 (2010).

8. Chen, X. et al. Mapping optogenetically-driven single-vessel fMRI with concurrent neuronal calcium recordings in the rat hippocampus. Nat. Commun. 10, 1-12 (2019).

9. Lebhardt, P., von Hohenberg, C. C., Weber-Fahr, W., Kelsch, W. \& Sartorius, A. Optogenetic fMRI in the mouse hippocampus: Hemodynamic response to brief glutamatergic stimuli. J. Cereb. Blood Flow Metab Off. J. Int. Soc. Cereb. Blood Flow Metab. 36, 629-638 (2016).

10. Liang, Z. et al. Mapping the functional network of medial prefrontal cortex by combining optogenetics and fMRI in Awake Rats. Neuroimage 117, 114-123 (2015).

11. Gerits, A. et al. Optogenetically-induced behavioral and functional network changes in primates. Curr. Biol. CB 22, $1722-1726$ (2012).

12. Weitz, A. J. \& Lee, J. H. Probing neural transplant networks in vivo with optogenetics and optogenetic fMRI. Stem Cells Int. 2016, $8612751(2016)$

13. Logothetis, N. K. What we can do and what we cannot do with fMRI. Nature 453, 869-878 (2008).

14. Macé, E. et al. Functional ultrasound imaging of the brain. Nat. Methods 8, 662-664 (2011).

15. Boido, D. et al. Mesoscopic and microscopic imaging of sensory responses in the same animal. Nat. Commun. 10, 1110 (2019).

16. Deffieux, T., Demene, C., Pernot, M. \& Tanter, M. Functional ultrasound neuroimaging: a review of the preclinical and clinical state of the art. Curr. Opin. Neurobiol. 50, 128-135 (2018).

17. Gesnik, M. et al. 3D functional ultrasound imaging of the cerebral visual system in rodents. Neuroimage 149, 267-274 (2017).

18. Macé, É. et al. Whole-brain functional ultrasound imaging reveals brain modules for visuomotor integration. Neuron 100, 12411251.e7 (2018)

19. Brunner, C. et al. A platform for brain-wide volumetric functional ultrasound imaging and analysis of circuit dynamics in Awake Mice. Neuron 108, 861-875.e7 (2020).

20. Sieu, L.-A. et al. EEG and functional ultrasound imaging in mobile rats. Nat. Methods 12, 831-834 (2015).

21. Bergel, A., Deffieux, T., Demené, C., Tanter, M. \& Cohen, I. Local hippocampal fast gamma rhythms precede brain-wide hyperemic patterns during spontaneous rodent REM sleep. Nat. Commun. 9, 1-12 (2018).

22. Dizeux, A. et al. Functional ultrasound imaging of the brain reveals propagation of task-related brain activity in behaving primates. Nat. Commun. 10, 1-9 (2019).

23. Blaize, K. et al. Functional ultrasound imaging of deep visual cortex in awake nonhuman primates. Proc. Natl. Acad. Sci. USA. 117, 14453-14463 (2020).

24. Norman, S. L. et al. Single-trial decoding of movement intentions using functional ultrasound neuroimaging. Neuron 109, 15541566.e4 (2021)

25. Rungta, R. L., Osmanski, B.-F., Boido, D., Tanter, M. \& Charpak, S. Light controls cerebral blood flow in naive animals. Nat. Commun. 8, 14191 (2017).

26. Sans-Dublanc, A. et al. Optogenetic fUSI for brain-wide mapping of neural activity mediating collicular-dependent behaviors. Neuron 576, 266 (2021).

27. Klapoetke, N. C. et al. Independent optical excitation of distinct neural populations. Nat. Methods 11, 338-346 (2014).

28. Mager, T. et al. High frequency neural spiking and auditory signaling by ultrafast red-shifted optogenetics. Nat. Commun. 9, 1750 (2018).

29. Marshel, J. H. et al. Cortical layer-specific critical dynamics triggering perception. Science 365, eaaw5202 (2019).

30. Gauvain, G. et al. Optogenetic therapy: high spatiotemporal resolution and pattern discrimination compatible with vision restoration in non-human primates. Commun. Biol. 4, 1-15 (2021).

31. GenSight Biologics. A Phase 1/2a, Open-Label, Non-Randomized, Dose-Escalation Study to Evaluate the Safety and Tolerability of GS030 in Subjects With Retinitis Pigmentosa. https://clinicaltrials.gov/ct2/show/NCT03326336 (2020).

32. Seabrook, T. A., Burbridge, T. J., Crair, M. C. \& Huberman, A. D. Architecture, function, and assembly of the mouse visual system. Annu. Rev. Neurosci. 40, 499-538 (2017).

33. Gao, Y.-R. et al. Time to wake up: studying neurovascular coupling and brain-wide circuit function in the un-anesthetized animal. Neuroimage 153, 382-398 (2017).

34. Yger, P. et al. A spike sorting toolbox for up to thousands of electrodes validated with ground truth recordings in vitro and in vivo. Elife 7, e34518 (2018).

35. Arenkiel, B. R. et al. In vivo light-induced activation of neural circuitry in transgenic mice expressing channelrhodopsin-2. Neuron 54, 205-218 (2007).

36. Stujenske, J. M., Spellman, T. \& Gordon, J. A. Modeling the spatiotemporal dynamics of light and heat propagation for in vivo optogenetics. Cell Rep. 12, 525-534 (2015).

37. Emiliani, V., Cohen, A. E., Deisseroth, K. \& Häusser, M. All-optical interrogation of neural circuits. J. Neurosci. 35, 13917-13926 (2015)

38. Chaigneau, E., Oheim, M., Audinat, E. \& Charpak, S. Two-photon imaging of capillary blood flow in olfactory bulb glomeruli. Proc. Natl. Acad. Sci. 100, 13081-13086 (2003).

39. Iadecola, C., Yang, G., Ebner, T. J. \& Chen, G. Local and propagated vascular responses evoked by focal synaptic activity in cerebellar cortex. J. Neurophysiol. 78, 651-659 (1997).

40. Iadecola, C. The neurovascular unit coming of age: a journey through neurovascular coupling in health and disease. Neuron $\mathbf{9 6}$, 17-42 (2017).

41. Kayser, C., Kim, M., Ugurbil, K., Kim, D.-S. \& König, P. A comparison of hemodynamic and neural responses in cat visual cortex using complex stimuli. Cereb. Cortex 14, 881-891 (2004)

42. O’Herron, P. et al. Neural correlates of single-vessel haemodynamic responses in vivo. Nature 534, 378-382 (2016) 
43. Chen, X., Wang, F., Fernandez, E. \& Roelfsema, P. R. Shape perception via a high-channel-count neuroprosthesis in monkey visual cortex. Science 370, 1191-1196 (2020).

44. Bi, A. et al. Ectopic expression of a microbial-type rhodopsin restores visual responses in mice with photoreceptor degeneration. Neuron 50, 23 (2006).

45. Lagali, P. et al. Light-activated channels targeted to ON bipolar cells restore visual function in retinal degeneration. Nat. Neurosci. 11, https://pubmed.ncbi.nlm.nih.gov/18432197/ (2008).

46. Gaub, B. M. et al. Restoration of visual function by expression of a light-gated mammalian ion channel in retinal ganglion cells or ON-bipolar cells. Proc. Natl. Acad. Sci. USA. 111, E5574-E5583 (2014).

47. Sengupta, A. et al. Red-shifted channelrhodopsin stimulation restores light responses in blind mice, macaque retina, and human retina. EMBO Mol. Med. 8, 1248-1264 (2016).

48. Chaffiol, A. et al. A new promoter allows optogenetic vision restoration with enhanced sensitivity in Macaque Retina. Mol. Ther. 25, 2546-2560 (2017).

49. Gauvain, G. et al. Optogenetic therapy: high spatiotemporal resolution and pattern recognition compatible with vision restoration in non-human primates. bioRxiv 2020.05.17.100230 (2020) https://doi.org/10.1101/2020.05.17.100230.

50. Khabou, H. et al. Noninvasive gene delivery to foveal cones for vision restoration. JCI Insight 3, (2018).

51. Jazayeri, M., Lindbloom-Brown, Z. \& Horwitz, G. D. Saccadic eye movements evoked by optogenetic activation of primate V1. Nat. Neurosci. 15, 1368-1370 (2012).

52. Ju, N., Jiang, R., Macknik, S. L., Martinez-Conde, S. \& Tang, S. Long-term all-optical interrogation of cortical neurons in awakebehaving nonhuman primates. PLoS Biol. 16, e2005839 (2018).

53. Choi, V. W., Asokan, A., Haberman, R. A. \& Samulski, R. J. Production of recombinant adeno-associated viral vectors. Curr. Protoc. Hum. Genet. Chapter 12, Unit 12.9 (2007).

54. Aurnhammer, C. et al. Universal real-time PCR for the detection and quantification of adeno-associated virus serotype 2-derived inverted terminal repeat sequences. Human Gene Ther. Methods 23, 18-28 (2012).

\section{Acknowledgements}

We thank Pierre Pouget, Frédéric Chavane, Brice Bathellier, Kévin Blaize, Sara Cadoni and Diep Nguyen for their useful discussions about the data. We thank Julie Dégardin for her help with surgery, Baptiste Lefebvre for assistance with spike sorting and Hicham Serroune for technical assistance with fUS imaging. This work was supported by the European Research Council (ERC) Synergy Grant Scheme (ERC Grant Agreement 610110), LabEx LIFESENSES (ANR-10-LABX-65) and IHU FOReSIGHT (ANR-18-IAHU-01).

\section{Author contributions}

M.P., S.P., J-A.S., M.T., G.G., and F.A. designed the study; M.P. and D.D. designed viral vectors; M.P. and W.D. produced viral vectors; M.P., G.L., A.R., R.G. and M.V. performed intracortical injections; M.P., G.L. and C.J. performed electrophysiological recordings and fUS acquisitions; G.L. performed histological experiments; M.P., U.F., A.C., G.G., F.A. and S.P. analyzed data; M.P., G.G. and F.A. constructed the figures; M.P., A.C., G.G., F.A. and S.P. wrote the manuscript.

\section{Competing interests}

M.T. is cofounder and S.P. and M.T. are shareholders of ICONEUS. D.D. and S.P. are consultants for Gensight Biologics. All other authors do not have any competing interest to disclose.

\section{Additional information}

Supplementary Information The online version contains supplementary material available at https:/doi.org/ 10.1038/s41598-021-91972-z.

Correspondence and requests for materials should be addressed to M.P.

Reprints and permissions information is available at www.nature.com/reprints.

Publisher's note Springer Nature remains neutral with regard to jurisdictional claims in published maps and institutional affiliations.

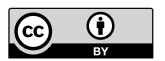

Open Access This article is licensed under a Creative Commons Attribution 4.0 International License, which permits use, sharing, adaptation, distribution and reproduction in any medium or format, as long as you give appropriate credit to the original author(s) and the source, provide a link to the Creative Commons licence, and indicate if changes were made. The images or other third party material in this article are included in the article's Creative Commons licence, unless indicated otherwise in a credit line to the material. If material is not included in the article's Creative Commons licence and your intended use is not permitted by statutory regulation or exceeds the permitted use, you will need to obtain permission directly from the copyright holder. To view a copy of this licence, visit http://creativecommons.org/licenses/by/4.0/.

(C) The Author(s) 2021 